\title{
a New Catalog of Silicate Carbon Stars
}

\author{
YounG-JoO KWOn AND KYUnG-WON SuH \\ Department of Astronomy and Space Science, Chungbuk National University, Cheongju 361-763, Korea \\ kwsuh@chungbuk.ac.kr
}

Received June 1, 2014; accepted June 17, 2014

\begin{abstract}
A silicate carbon star is a carbon star which shows circumstellar silicate dust features. We collect a sample of 44 silicate carbon stars from the literature and investigate the validity of the classification. For some objects, it is uncertain whether the central star is a carbon star. We confirm that 29 objects are verified silicate carbon stars. We classify the confirmed objects into three subclasses based on the evolution phase of the central star. To investigate the effect of the chemical transition phase from $\mathrm{O}$ to $\mathrm{C}$, we use the radiative transfer models for the detached silicate dust shells. The spectral energy distributions and the infrared two-color diagrams of the silicate carbon stars are compared with the theoretical model results. For the chemical transition model without considering the effect of a disk, we find that the life time of the silicate feature is about 50 to 400 years depending on the initial dust optical depth.
\end{abstract}

Key words: stars: AGB stars - infrared: stars — circumstellar matter - masers - dust: extinction

\section{INTRODUCTION}

A silicate carbon star (hereafter; SCS) is a carbon star which shows circumstellar silicate dust features. They were discovered by the IRAS observations (LittleMarenin 1986; Willems \& de Jong 1986). So far, about 35 SCSs have been identified in our Galaxy (e.g., Suh \& Kwon 2011).

As stars evolve into the thermal pulsing asymptotic giant branch (AGB) phase, the abundances of some elements in the stellar atmosphere may change by the episodic third dredge-up process after each thermal pulse. The stepwise increase of the carbon abundance is related to the formation of a carbon star because the $\mathrm{C} / \mathrm{O}$ abundance ratio can change from $\mathrm{C} / \mathrm{O}<1$ to $\mathrm{C} / \mathrm{O}$ $>1$ (e.g., Busso et al. 1999). These stars may experience the $\mathrm{S}$ star phase before they become carbon stars when the $\mathrm{C} / \mathrm{O}$ ratio is close to 1 . When AGB stars of intermediate mass range go through carbon dredge-up processes, the abundance of carbon becomes larger than that of oxygen, O-rich dust grain formation ceases, and the stars become visual carbon stars. After that phase, C-rich dust grains start forming (Iben 1981; Chan \& Kwok 1990; Groenewegen et al. 1995; Suh 2000).

Willems \& de Jong (1986) and Chan \& Kwok (1991) suggested that SCSs are in transition from O-rich AGB stars to carbon stars. Many of the SCSs are fairly well fitted with a simple detached silicate dust shell model. However, this scenario could be unlikely because the time scale for such a transitional object to be observed as a SCS is predicted to be too short.

A more promising scenario suggests that SCSs have a low-luminosity companion, possibly a dwarf star (e.g., Morris 1987; Lloyd Evans 1990; Yamamura et al. 2000). A relatively long-lived disk of silicate dust is supposed to be formed when the primary was still an O-rich AGB

Corresponding AUthor: K.-W. Suh star. When the primary star was an M-type giant and O-rich materials were supposed to be used to form a circumbinary (or circum-companion) disk. The disk may serve as a reservoir of O-rich material even after the star undergoes thermal pulses and evolves into a carbon star.

For a large sample of AGB stars, infrared observational data are available from the Infrared Astronomical Satellite (IRAS), Infrared Space Observatory (ISO), Midcourse Space Experiment (MSX), USNOB1.0 (Monet et al. 2003), Two-Micron All-Sky Survey (2MASS), Wide-field Infrared Survey Explorer (WISE) and AKARI. The IRAS point source catalog (PSC) version 2.1 contains useful photometric data in four bands $(12,25,50$ and $100 \mu \mathrm{m}$ ). The MSX (Egan et al. 2003) PSC provides useful photometric data in 8.28, 12.13, 14.65, $21.34 \mu \mathrm{m}$ wavelength bands. The 2MASS (Cutri et al. 2003) PSC contains accurate positions and fluxes for about 470 million stars and other unresolved objects in $J(1.25 \mu \mathrm{m}), H(1.65 \mu \mathrm{m})$ and $K(2.17 \mu \mathrm{m})$ bands. The WISE (Wright et al. 2010) surveyed the entire sky with much higher sensitivity than previous missions. The AKARI space telescope (Murakami et al. 2007) made an all sky survey with the infrared camera (IRC) and far infrared surveyor (FIS). We may use the AKARI PSC data in two bands ( 9 and $18 \mu \mathrm{m}$ ) obtained by the IRC and the bright-source catalogue (BSC) data in four bands $(65,90,140$ and $160 \mu \mathrm{m})$ obtained by the FIS.

The IRAS Low Resolution Spectrograph (LRS; $\lambda=$ $8-22 \mu \mathrm{m}$ ) data are useful to identify important dust features of SCSs. Kwok et al. (1997) used IRAS LRS to identify the class $\mathrm{E}$ (the $10 \mu \mathrm{m}$ silicate feature in emission) and class A (the $10 \mu \mathrm{m}$ silicate feature in absorption) objects. The ISO Short Wavelength Spectrometer (SWS; $\lambda=2.4-45.4 \mu \mathrm{m}$ ) and Spitzer Infrared Spectrograph (IRS; $\lambda=5.2-38 \mu \mathrm{m}$ ) provided high res- 
olution spectra for some SCSs.

In this paper, we present a new catalog of SCSs. We confirm the C-rich nature of central stars and O-rich nature of circumstellar envelopes for the sample SCSs. To investigate the effect of the chemical transition phase from $\mathrm{O}$ to $\mathrm{C}$, we use radiative transfer models for the detached silicate dust shells. We compare the spectral energy distributions and infrared two color diagrams of the SCSs with the theoretical model results and estimate the evolutionary time scales for SCSs.

\section{Sample Collection and Confirmation}

Suh \& Kwon (2011, hereafter SK11) listed 35 SCSs. We have used the 35 objects in SK11 to construct the initial sample of stars to be verified in this paper.

Chen \& Shan (2011, hereafter CS11) presented 5 new SCSs which were verified from 17 new candidate objects as well as 34 previously known SCSs. The 12 candidate objects which were not verified to be SCSs by CS11 are regarded as unconfirmed objects (see Section 3 ). The two objects (IRAS 06176-1036 and 13198-6224) listed in CS11 as previously known SCSs were not listed in SK11. We add those 7 objects to the sample stars (the 5 new SCSs and 2 previously known objects presented in CS11).

We add two more objects: IRAS $19163+2745$ (EP Lyr) and IRAS 20451+4552 (CY Cyg). EP Lyr is a post-AGB star which shows both polycyclic aromatic hydrocarbon (PAH) and crystalline silicate features like the Red Rectangle (Gielen et al. 2008, 2009). CY Cyg is a SC type star with silicate emission feature. According to Kwok et al. (1997), there are 4 SC type stars (W Cas, VX Aql, S Lyr and CY Cyg) and a CS type star (IRAS 09083-5158) which are in LRS class E.

Finally, the 44 objects are selected for the sample of SCSs to be verified in this paper and they are listed in Table 1. Because the AKARI PSC measures the flux of the peak emission with a much smaller aperture (9 arcsec beam size) than IRAS PSC, we use the AKARI PSC position for further identification. For the 44 objects, we cross-identify the AKARI PSC counterpart by finding the nearest source within $30^{\prime \prime}$ using the position information of the IRAS PSC (version 2.1). For each sample star, we have found an AKARI source within $30^{\prime \prime}$ except for IRAS 16070-4359 which has two AKARI sources. For the IRAS 16070-4359, the counterpart is selected by comparing the flux data with the IRAS PSC.

We use the counterpart of the General Catalog of Galactic Carbon stars (Alksnis et al. 2001, hereafter CGCS) based on the position information of the AKARI PSC as well as the available spectral evidences in optical, infrared and radio regions.

In Table 1, we present the IRAS PSC number, AKARI PSC counterpart, position information from the AKARI PSC counterpart, CGCS counterpart, and IRAS LRS class. For the 44 sample objects, we investigate the validity of the classification. To confirm the nature of the SCS, we investigate the signatures of the C-rich nature of central star and O-rich nature of circumstellar envelope.

\subsection{The CGCS Counterparts}

We identify the CGCS counterparts for which the position difference between the AKARI PSC and CGCS is less than $2^{\prime}$. For the 44 sample stars, we find that 35 objects have the CGCS counterparts and they are listed in Table 1 . The position differences $(\Delta \mathrm{r})$ between the AKARI PSC and CGCS are also listed in Table 1.

For the 35 CGCS counterparts, only 3 sources have position differences larger than $30^{\prime \prime}$ (IRAS 12364-6539, 16070-4359 and 18252-1011). For the three sources, the AKARI, MSX and 2MASS counterparts based on the IRAS PSC and CGCS positions are different. These objects seem to be different sources. We mark those objects with the asterisk symbol in Table 1 . We list the 3 objects as unconfirmed objects in Table 2 . These 3 stars with large position differences need more reliable observation data to confirm their nature.

The O-rich nature of the outer shells can be easily recognized by IRAS LRS class by Kwok et al. (1997). Among the 35 stars, 33 stars with silicate emission features are classified as E class, and 2 stars (IRAS 07204-1032 and 12364-6539) as F class. For IRAS 07204-1032, Engels (1994) detected $\mathrm{H}_{2} \mathrm{O}$ maser. $\mathrm{H}_{2} \mathrm{O}$ maser occurs only in the dusty O-rich environments. The spectral type of the last one, IRAS $12364-6539$, is M9 according to Skiff (2014). Note that this is one of the unconfirmed objects with the large position difference between the AKARI and CGCS.

\subsection{The Sample Stars without CGCS Couterparts}

As mentioned in Section 2.1, 9 stars do not have CGCS counterparts within $3^{\prime}$. We check these stars using the available optical and infrared spectral information from the literature.

$\mathrm{C}_{2} \mathrm{H}_{2}+\mathrm{HCN}$ absorption features around $3.0 \mu \mathrm{m}$ observed by the ISO SWS are clear evidences of the central carbon star. There are obvious $\mathrm{C}_{2} \mathrm{H}_{2}+\mathrm{HCN}$ absorption features for three stars: IRAS $03201+5459$, 09425-6040 and 19111+2555 (e.g., Molster et al. 2001). These three stars show amorphous or crystalline silicate features.

IRAS 06176-1036 and 19163+2745 (Red Rectangle and EP Lyr) show the PAH and crystalline silicate features detected by ISO-SWS or Spitzer-IRS (e.g., Gielen et al. 2008, 2009).

Drew et al. (2005) observed the optical spectrum toward IRAS $22146+6111$ and identified this star as a carbon star. IRAS 20451+4552 (CY Cyg) is classified as an SC star by Keenan \& Boeshaar (1980). Kwok et al. (1997) classified IRAS $21135+5200$ as a carbon star. All the 3 stars show a silicate emission feature at $10 \mu \mathrm{m}$ detected from the IRAS LRS.

However, the remaining one object, IRAS $21444+5053$ has no direct evidence of a carbon star. We list it as an unconfirmed object in Table 2. We present a detailed discussion for all of the unconfirmed objects in Section 3. 
Table 1

Sample objects for SCSs (44 objects)

\begin{tabular}{|c|c|c|c|c|c|c|c|c|}
\hline IRAS PSC & Other Name & AKARI PSC & R.A. (2000) & Dec. $(2000)$ & CGCS & $\Delta \mathrm{r}(\operatorname{arcsec})$ & LRS & Ref. \\
\hline $00519+5817$ & W Cas & $0054539+583349$ & 13.72478 & 58.56379 & 136 & 0.72 & $\mathrm{E}$ & SK11; CS11 \\
\hline $01373+4000$ & FBS $0137+400$ & $0140206+401518$ & 25.08616 & 40.25515 & 6019 & 10.44 & $\mathrm{E}$ & SK11; CS11 \\
\hline $03201+5459$ & - & $0323593+551014$ & 50.99747 & 55.17075 & - & - & $\mathrm{U}$ & SK11; CS11 \\
\hline $06017+1011$ & NSV 2814 & $0604313+101056$ & 91.13083 & 10.18243 & 1158 & 28.74 & $\mathrm{E}$ & SK11; CS11 \\
\hline $06176-1036$ & Red rectangle & $0619582-103814$ & 94.99252 & -10.63743 & - & - & $\mathrm{P}$ & CS11 \\
\hline $06194+1635$ & - & $0622213+163423$ & 95.58881 & 16.57326 & 6139 & 3.36 & $\mathrm{E}$ & SK11; CS11 \\
\hline $07179+2505$ & BM Gem & $0720590+245958$ & 110.24605 & 24.99947 & 1653 & 0.72 & $\mathrm{E}$ & SK11; CS11 \\
\hline $07204-1032$ & - & 0722469-103839 & 110.69561 & -10.64440 & 1682 & 2.94 & $\mathrm{~F}$ & SK11 \\
\hline $08002-3803$ & Hen 38 & $0802050-381151$ & 120.52102 & -38.19763 & 2011 & 3 & $\mathrm{E}$ & SK11; CS11 \\
\hline $08577-6035$ & Mc97 2-11 & $0858542-604735$ & 134.72621 & -60.79326 & 2406 & 17.58 & $\mathrm{E}$ & SK11; CS11 \\
\hline $09083-5158$ & - & $0909592-521020$ & 137.49690 & -52.17228 & 2445 & 39.54 & $\mathrm{E}$ & SK11; CS11 \\
\hline $09411-5933$ & - & $0942361-594737$ & 145.65059 & -59.79373 & 6337 & 1.98 & $\mathrm{E}$ & SK11; CS11 \\
\hline $09425-6040$ & - & $0944017-605425$ & 146.00731 & -60.90697 & - & - & $\mathrm{U}$ & SK11; CS11 \\
\hline $10171-6205$ & - & $1018503-622101$ & 154.70996 & -62.35050 & 6359 & 0.66 & $\mathrm{E}$ & SK11; CS11 \\
\hline $11048-6046$ & - & 1106546-610301 & 166.72757 & -61.05033 & 2957 & 24.84 & $\mathrm{E}$ & SK11; CS11 \\
\hline $11311-6355$ & - & $1133246-641158$ & 173.35264 & -64.19952 & 6450 & 2.34 & $\mathrm{E}$ & SK11; CS11 \\
\hline $12364-6539$ & - & $1239236-655603$ & 189.84839 & -65.93439 & $3263^{*}$ & 50.52 & $\mathrm{~F}$ & SK11 \\
\hline $12569-6105$ & - & $1300043-612118$ & 195.01814 & -61.35526 & 6503 & 2.52 & $\mathrm{E}$ & SK11; CS11 \\
\hline $13198-6224$ & RAFGL 4890S & $1323088-623954$ & 200.78697 & -62.66526 & 6526 & 14.16 & $\mathrm{E}$ & CS11 \\
\hline $14286-4706$ & - & $1431574-471943$ & 217.98948 & -47.32878 & 6559 & 1.32 & $\mathrm{E}$ & SK11; CS11 \\
\hline $15575-5238$ & - & $1601205-524649$ & 240.33542 & -52.78041 & 6610 & 10.02 & $\mathrm{E}$ & CS11 \\
\hline $16001-4851$ & - & $1603478-485915$ & 240.94942 & -48.98765 & 6612 & 1.68 & $\mathrm{E}$ & SK11; CS11 \\
\hline $16070-4359$ & - & $1610358-440654$ & 242.64945 & -44.11517 & $3668^{*}$ & 101.1 & $\mathrm{E}$ & CS11 \\
\hline $16103-4929$ & - & $1614054-493658$ & 243.52267 & -49.61615 & 6624 & 1.62 & $\mathrm{E}$ & SK11; CS11 \\
\hline $16328-4656$ & - & $1636291-470258$ & 249.12155 & -47.04954 & 6650 & 5.1 & $\mathrm{E}$ & SK11; CS11 \\
\hline $17291-3401$ & - & $1732281-340313$ & 263.11734 & -34.05384 & 3841 & 4.32 & $\mathrm{E}$ & SK11 \\
\hline $18006-3213$ & FJF 270 & $1803527-321259$ & 270.96992 & -32.21660 & 3935 & 15.3 & $\mathrm{E}$ & SK11; CS11 \\
\hline $18252-1011$ & - & 1828010-100917 & 277.00444 & -10.15479 & $4015^{*}$ & 106.98 & $\mathrm{E}$ & SK11; CS11 \\
\hline $18575-0139$ & VX Aql & $1900096-013458$ & 285.04029 & -1.58291 & 4152 & 2.94 & $\mathrm{E}$ & SK11; CS11 \\
\hline $19111+2555$ & S Lyr & $1913117+260027$ & 288.29915 & 26.00775 & - & - & $\mathrm{E}$ & SK11; CS11 \\
\hline $19139+5412$ & NC 83 & $1915015+541729$ & 288.75646 & 54.29144 & 4222 & 3.84 & $\mathrm{E}$ & SK11; CS11 \\
\hline $19163+2745$ & EP Lyr & $1918195+275103$ & 289.58154 & 27.85093 & - & - & - & New \\
\hline $19451+2856$ & NSV 12406 & $1947131+290406$ & 296.80466 & 29.06848 & 4476 & 1.56 & $\mathrm{E}$ & SK11; CS11 \\
\hline $19583-0730$ & V1468 Aql & $2001051-072152$ & 300.27158 & -7.36445 & 4595 & 0.3 & $\mathrm{E}$ & SK11; CS11 \\
\hline $20092+3557$ & V429 Cyg & $2011061+360648$ & 302.77579 & 36.11347 & 4729 & 1.86 & $\mathrm{E}$ & SK11; CS11 \\
\hline $20350+5954$ & V778 Cyg & $2036073+600525$ & 309.03083 & 60.09049 & 4923 & 5.28 & $\mathrm{E}$ & SK11; CS11 \\
\hline $20451+4552$ & CY Cyg & $2046503+460306$ & 311.70962 & 46.05176 & - & - & $\mathrm{E}$ & New \\
\hline $20476+4422$ & - & $2049250+443348$ & 312.35417 & 44.56353 & 4993 & 2.94 & $\mathrm{E}$ & SK11; CS11 \\
\hline $21135+5200$ & - & $2115081+521249$ & 318.78392 & 52.21375 & - & - & $\mathrm{E}$ & SK11; CS11 \\
\hline $21444+5053$ & - & $2146149+510729$ & 326.56247 & 51.12478 & - & - & $\mathrm{E}$ & CS11 \\
\hline $21566+5309$ & MQ Cyg & $2158261+532412$ & 329.60903 & 53.40343 & 5526 & 1.74 & $\mathrm{E}$ & SK11; CS11 \\
\hline $22146+6111$ & - & $2216197+612621$ & 334.08234 & 61.43923 & - & - & $\mathrm{E}$ & CS11 \\
\hline $23138+6204$ & NSV 14477 & $2316023+622118$ & 349.00976 & 62.35502 & 6912 & 1.98 & $\mathrm{E}$ & CS11 \\
\hline $23176+4658$ & EU And & $2319589+471434$ & 349.99544 & 47.24282 & 5848 & 4.38 & $\mathrm{E}$ & SK11; CS11 \\
\hline
\end{tabular}

SK11 = Suh \& Kwon (2011); CS11 = Chen \& Shan (2011). ${ }^{*}$ The object seems to be a different source (See Section 3). 


\subsection{Two-Color Diagrams}

For some sample stars having the CGCS number, they are classified to be carbon stars by the method based on the color index. This method classifies the stars based on their location in a two-color diagram (2CD). Epchtein et al. $(1987,1990)$ showed that the 2CD using [12]-[25] versus $K-L$ allows to distinguish carbon stars from O-rich stars with some reliability. In the $2 \mathrm{CD}$ using [12]-[25] versus $K-L$, carbon stars are located in the lower region and O-rich stars appear in the upper region. However, it is well known that the position of an AGB star on a $2 \mathrm{CD}$ varies widely depending on the phase of pulsation (e.g., Suh 2004). The variation of the positions on 2CDs could lead to mis-classifications especially for the stars near the border.

Therefore, the color index method is not sufficient to reveal the C-rich nature of the central star. For the 10 objects, for which the C-rich nature is solely determined from the 2CD method, we need further verifications. We list these as unconfirmed objects in Table 2 .

\section{UNCONFIRMED OBJECTS}

Through the investigations described in Section 2, we confirm that 29 objects are real SCSs. But for 15 objects, it is not clear whether the central star is a real carbon star. We list the 15 unconfirmed objects in Table 2 .

CS11 presented 12 candidate objects ( 5 candidates in table $2 ; 7$ probable candidates in Table 3 ) which were not verified to be real SCSs. In Table 3, we list the 12 candidate objects presented by CS11 which can also be regarded as unconfirmed objects.

For all of the 15 unconfirmed objects, the silicate emission feature at $10 \mu \mathrm{m}$ is detected in the IRAS LRS spectrum. Kwok et al. (1997) classified them as the IRAS LRS class E.

IRAS 06194+1635 (CGCS 6139): Guglelmo et al. (1993) identified it as a carbon star based on the 2CD using [12]-[25] versus $K-L$. Althogh the object is in the region of carbon stars, its C-rich nature is not clear (see Section 2.3).

IRAS 09411-5933 (CGCS 6337): This star was identified to be a carbon star by Fouque et al. (1992) using the [12]-[25] and $K-L$ color indices. However, Skiff (2014) identified its spectral type as M2/3. OH maser emission at $1612 \mathrm{MHz}$ was not detected in $\mathrm{OH}$ maser survey of te Lintel Hekkert (1991).

IRAS 11311-6355 (CGCS 6450): Guglielmo et al. (1993) first identified it as a carbon star from the 2CD using [12]-[25] versus $K-L$. Kwok et al. (1997) classified this star as LRS class E with self-absorption. As mentioned above, the color index method is not enough for revealing the C-rich nature of the central star.

IRAS 12364-6539: This star has a large position difference from CGCS $3264\left(\Delta \mathrm{r}=51^{\prime \prime}\right)$. Skiff (2014) identified the spectral type as M9. The AKARI, MSX and 2MASS counterparts based on the IRAS PSC position and CGCS position are different. IRAS 12364-6539 seems to be an O-rich star rather than an SCS.
IRAS 12569-6105 (CGCS 6503): Epchtein et al. (1990) classified it as a carbon star using a 2CD. MacConnell (2000) and Skiff (2014) classified this star as an $\mathrm{S}$ star.

IRAS 13198-6224 (CGCS 6610): Aaronson et al. (1989) classified it as a carbon star, but Lloyd Evans (1991) argued that the spectral type is M2.

IRAS 14286-4706 (CGCS 6559): Fouque et al. (1992) firstly identified this object as a carbon star using the [12]-[25] versus $K-L 2 \mathrm{CD}$. It is seen near the border region between $\mathrm{O}$-rich AGB stars and carbon stars on the $2 \mathrm{CD}$.

IRAS 16001-4851 (CGCS 6612): Epchtein et al. (1990) identified it as a carbon star using the color index method. Its C-rich nature is not clear

IRAS 16070-4359: Guglielmo et al. (1993) identified it as a carbon star from the 2CD using [12]-[25] versus $K-L$. However, this star has a large position difference between the AKARI and CGCS positions $\left(\Delta \mathrm{r}=101^{\prime \prime}\right)$. The AKARI, MSX and 2MASS counterparts based on the IRAS PSC position and CGCS position are different. Therefore, IRAS 16070-4359 and CGCS 3668 seem to be different objects.

IRAS 16103-4929 (CGCS 6624): Epchtein et al. (1990) classified this star as carbon star based on the color index method. Skiff (2014) revealed that the spectral type is M4,M6. Therefore, this star could be a O-rich star with large interstellar extinction.

IRAS 16328-4656 (CGCS 6650): Although Epchtein et al. (1987) first identified this star as a carbon star based on the color index method, it is necessary to confirm the C-rich nature of the central star.

IRAS 17291-3401 (CGCS 3841): Chan \& Kwok (1991) first identified it as an SCS based on the CGCS. However, MacConnell (2006) reported it as a M3 star by the NIR spectral observation. This star seems to be misclassified as a carbon star.

IRAS 18252-1011: The C-rich nature is not certain. This star has a large position difference between the AKARI and CGCS positions $\left(\Delta \mathrm{r}=107^{\prime \prime}\right)$. The AKARI, MSX and 2MASS counterparts based on the IRAS PSC position and CGCS position are different.

IRAS 20476+4422 (CGCS 4993): Although this object has a CGCS counterpart and shows the silicate emission feature at $10 \mu \mathrm{m}$, its C-rich nature is not clear.

IRAS 21444+5053: This object is not listed in CGCS and there is no available spectral information to confirm its nature. Jiang et al. (1997) identified this star as a carbon star based on the J-H versus $\mathrm{H}-\mathrm{K} 2 \mathrm{CD}$. However, it was classified as an O-rich star based on the color index method by Guglielmo et al. (1997). Groenewegen et al. (2002) detected CO emission but did not detect HCN and $\mathrm{SiO}$ emission. For this object, further investigations are necessary.

\section{Subclasses of SCSS}

For the 29 confirmed SCSs, we classify them into three subclasses based on the evolutionary stage of the central star. The objects in subclass 1 are five SC/CS 
Table 2

The 15 unconfirmed objects which were classified to be SCSs

\begin{tabular}{llllll}
\hline IRAS PSC & IRAS LRS & CGCS & Spectrum & 2CD & Position \\
\hline $06194+1635$ & $\mathrm{E}$ & 6139 & - & Color based $^{1)}$ & - \\
$09411-5933$ & $\mathrm{E}$ & 6337 & $\mathrm{M} 2 / 3^{a)}$ & Color based $^{2)}$ & - \\
$11311-6355$ & $\mathrm{E}$ & 6450 & - & Color based $^{1)}$ & - \\
$12364-6539$ & $\mathrm{~F}$ & $3263^{*}$ & $\mathrm{M} 9^{a)}$ & - & $\Delta \mathrm{r}=51^{\prime \prime}$ \\
$12569-6105$ & $\mathrm{E}$ & 6503 & $\mathrm{~S}^{b)}$ & Color based $^{3)}$ & - \\
$13198-6224$ & $\mathrm{E}$ & 6526 & $\mathrm{C}^{c)} ; \mathrm{M} 2^{d)}$ & - & - \\
$14286-4706$ & $\mathrm{E}$ & 6559 & - & Color based $^{2)}$ & - \\
$16001-4851$ & $\mathrm{E}$ & 6612 & - & Color based $^{3)}$ & - \\
$16070-4359$ & $\mathrm{E}$ & $3668^{*}$ & - & Color based $^{1)}$ & $\Delta \mathrm{r}=101^{\prime \prime}$ \\
$16103-4929$ & $\mathrm{E}$ & 6624 & $\mathrm{M} 4, \mathrm{M} 6^{a)}$ & Color based $^{3)}$ & - \\
$16328-4656$ & $\mathrm{E}$ & 6650 & - & Color based $^{3)}$ & - \\
$17291-3401$ & $\mathrm{E}$ & 3841 & $\mathrm{M} 3^{e)}$ & - & - \\
$18252-1011$ & $\mathrm{E}$ & $4015^{*}$ & - & - & $\Delta \mathrm{r}=107^{\prime \prime}$ \\
$20476+4422$ & $\mathrm{E}$ & 4993 & - & - & - \\
$21444+5053$ & $\mathrm{E}$ & - & - & Color based $^{4)}$ & - \\
\hline
\end{tabular}

Spectrum: a) Skiff (2014); b) MacConnell (2000); c) Aaronson et al. (1989); d) Lloyd Evans (1991); e) MacConnell (2006); f) Keenan \& Boeshaar (1980)

2CD: 1) Guglielmo et al. (1993); 2) Fouque et al. (1992); 3) Epchtein et al. (1990); 4) Jiang et al. (1997).

${ }^{*}$ The marked object looks to be a different source. See Section 3.

Table 3

The 12 candidate objects which were not verified to be SCSs by Chen \& Shan (2011)

\begin{tabular}{cccccc}
\hline IRAS PSC & AKARI PSC & Note & IRAS PSC & AKARI PSC \\
\hline $06462-4157$ & $0647513-420109$ & candidate & $16304-3831$ & $1633527-383752$ & Probable candidate \\
$15488-4928$ & $1552301-493723$ & candidate & $16469-4753$ & $1650398-475856$ & Probable candidate \\
$17354-3455$ & $17384548-3457177^{a}$ & candidate & $17599-4556$ & $1803383-455637$ & Probable candidate \\
$17515-2407$ & $1754366-240752$ & candidate & $18038-1614$ & $1806443-161335$ & Probable candidate \\
$18240-3006$ & $1827159-300439$ & candidate & $18559+0435$ & $1858242+043954$ & Probable candidate \\
$12195-5527$ & $1222168-554352$ & Probable candidate & $19270+2239$ & $1929101+224542$ & Probable candidate \\
\hline
\end{tabular}

${ }^{a}$ The 2MASS counterpart. There is no AKARI counterpart for the object.

stars showing weak silicate emission features. The objects in subclass 2 are 22 carbon stars showing silicate features. In subclass 3, there are two C-rich post-AGB stars showing crystalline silicate features. We list them in Table 4.

The general catalogue of variable stars (GCVS: Samus et al. 2011) contains the list of LPVs for different variable types. LPVs in AGB phase are classified according to the amplitude and regularity of the period into Miras (M), semi-regulars (SR), irregular (L), and RV Tauri type (RV) variables. The GCVS variability type is also listed in Table 4.

In Figure 1, we present the relative spectral energy distributions (SEDs) for the 29 objects. The SEDs show the observational data of USNO-B, 2MASS, WISE, AKARI, IRAS and ISO which cover the wavelength range from $0.9 \mu \mathrm{m}$ to $100 \mu \mathrm{m}$. They show the relative fluxes based on the $K$ band data.

The five objects in subclass 1 are SC/CS stars showing weak silicate emission features. The SC/CS stars (Keenan \& Boeshaar 1980) form a continuous spectral sequence intermediate between the $\mathrm{S}$ and $\mathrm{C}$ stars. They show very strong sodium D-lines, and strong $\mathrm{CN}$ bands, and either weak $\mathrm{ZrO}$ bands (SC stars) or $\mathrm{C}_{2}$ bands
(CS stars). The molecular abundances indicate a $\mathrm{C} / \mathrm{O}$ number ratio very close to unity, so that $\mathrm{CO}$ formation leaves little $\mathrm{C}$ or $\mathrm{O}$ for the other molecules. SC or CS stars are likely to be in a transitional phase from an $\mathrm{S}$ star to a carbon star. For lower metallicity, stars may go from being O-rich to being carbon stars without any Stype star phase, depending on the dredge-up efficiency and the initial mass of the star (e.g., Iben 1981; Chan and Kwok 1990; Groenewegen et al. 1995). Generally, dust formation in envelopes of $\mathrm{S}$ stars is less efficient because of the lack of free $\mathrm{O}$ or $\mathrm{C}$ to form dust. This could easily explain the weak silicate emission features generally observed in this subclass.

Most stars in subclass 2 show silicate emission features. However, IRAS $03201+5459$ shows the silicate absorption feature at $10 \mu \mathrm{m}$. And crystalline silicate emission features are observed from IRAS 09425-6040. 11 objects in this subclass are J-type carbon stars (see, e.g., Chen et al. 2007). Groenewegen et al. (1995) argued that the $\mathrm{C} / \mathrm{O}$ abundance ratio for the star of low initial masses increases in a few and rather larger steps and they probably skip the intermediate state of an $\mathrm{S}$ star $(\mathrm{C} / \mathrm{O} \sim 1)$ in most cases. The stars in subclass 2 could be in this case. The rich silicate features (see Sec- 
Table 4

A new catalog of silicate-carbon stars (29 objects)

\begin{tabular}{|c|c|c|c|c|c|c|}
\hline IRAS & Other Name & CGCS & LRS & GCVS & Spectrum & Note \\
\hline \multicolumn{7}{|c|}{ 1) SC/CS stars with silicate features } \\
\hline $00519+5817$ & W Cas & 136 & $\mathrm{E}$ & $\mathrm{M}$ & $\mathrm{SC}^{1), 14)}$ & $\mathrm{C}_{2} \mathrm{H}_{2}+\mathrm{HCN}$ \\
\hline $09083-5158$ & - & 2445 & $\mathrm{E}$ & - & $\mathrm{CS}^{2)}$ & \\
\hline $18575-0139$ & VX Aql & 4152 & $\mathrm{E}$ & M: & $\mathrm{SC} 8^{1)}$ & \\
\hline $19111+2555$ & S Lyr & - & $\mathrm{E}$ & M & $\mathrm{SCe}^{3)}$ & $\mathrm{C}_{2} \mathrm{H}_{2}+\mathrm{HCN}$ \\
\hline $20451+4552$ & CY Cyg & - & $\mathrm{E}$ & LB & $\mathrm{SC} 2^{1)}$ & \\
\hline \multicolumn{7}{|c|}{ 2) Carbon stars with silicate features (22 objects) } \\
\hline $01373+4000$ & FBS $0137+400$ & 6019 & $\mathrm{E}$ & - & $\mathrm{C}(\mathrm{N})^{2)}$ & self-absorption \\
\hline $03201+5459$ & - & - & $\mathrm{U}$ & - & - & $\mathrm{C}_{2} \mathrm{H}_{2}+\mathrm{HCN}$; silicate absorption \\
\hline $06017+1011$ & - & 1158 & $\mathrm{E}$ & - & $\mathrm{C} 4,5 \mathrm{~J}^{4)}$ & $\mathrm{H}_{2} \mathrm{O}$ maser \\
\hline $07179+2505$ & BM Gem & 1653 & $\mathrm{E}$ & $\mathrm{SRb}$ & $\mathrm{C} 5,4 \mathrm{~J}^{4)}$ & \\
\hline $07204-1032$ & - & 1682 & $\mathrm{~F}$ & - & $\mathrm{C} 4,5 \mathrm{~J}^{2)}$ & $\mathrm{H}_{2} \mathrm{O}$ maser \\
\hline $08002-3803$ & Hen 38 & 2011 & $\mathrm{E}$ & - & $\mathrm{C} 3,5 \mathrm{~J}^{4)}$ & \\
\hline $08577-6035$ & Mc79 2-11 & 2406 & $\mathrm{E}$ & - & $\mathrm{C} 4,5 \mathrm{~J}^{4)}$ & $\mathrm{H}_{2} \mathrm{O}$ maser \\
\hline $09425-6040$ & - & - & $\mathrm{U}$ & - & - & $\mathrm{C}_{2} \mathrm{H}_{2}+\mathrm{HCN}$; crystalline silicate \\
\hline $11048-6046$ & - & - & $\mathrm{E}$ & - & $C::^{13)}$ & \\
\hline $10171-6205$ & - & 6359 & $\mathrm{E}$ & - & $\mathrm{C} 4,4 \mathrm{~J}^{2)}$ & \\
\hline $15575-5238$ & - & 6610 & $\mathrm{E}$ & - & $\mathrm{C}^{12)}$ & \\
\hline $18006-3213$ & FJF 270 & 3935 & $\mathrm{E}$ & - & $\mathrm{C} 5,5 \mathrm{~J}^{4)}$ & $\mathrm{OH}$ maser \\
\hline $19139+5412$ & NC 83 & 4222 & $\mathrm{E}$ & $\mathrm{SR}$ & $C^{5)}$ & \\
\hline $19451+2856$ & NSV 12406 & 4476 & $\mathrm{E}$ & - & $\mathrm{C}^{8)}$ & \\
\hline $19583-0730$ & V1468 Aql & 4595 & $\mathrm{E}$ & $\mathrm{Lb}$ & $\mathrm{C} 5,5 \mathrm{~J}^{4)}$ & \\
\hline $20092+3557$ & V429 Cyg & 4729 & $\mathrm{E}$ & SRa & $\mathrm{C} 5,4^{8)}$ & \\
\hline $20350+5954$ & V778 Cyg & 4923 & $\mathrm{E}$ & $\mathrm{Lb}$ & $\mathrm{C} 4,5 \mathrm{~J}^{4)}$ & $\mathrm{OH} / \mathrm{H}_{2} \mathrm{O}$ maser \\
\hline $21135+5200$ & 12.84 & - & $\mathrm{E}$ & - & $\mathrm{C}:{ }^{13)}$ & \\
\hline $21566+5309$ & MQ Cyg & 5526 & $\mathrm{E}$ & M: & $\mathrm{C}(\mathrm{J})^{7)}$ & \\
\hline $22146+6111$ & - & - & $\mathrm{E}$ & - & $\mathrm{C}^{9)}$ & self-absorption \\
\hline $23138+6204$ & NSV 14477 & 6912 & $\mathrm{E}$ & - & $\mathrm{C}^{4)}$ & \\
\hline $23176+4658$ & EU And & 5848 & $\mathrm{E}$ & Lb: & $\mathrm{C} 4,4 \mathrm{~J}^{4)}$ & $\mathrm{H}_{2} \mathrm{O}$ maser \\
\hline \multicolumn{7}{|c|}{ 3) C-rich post-AGB stars with silicate features } \\
\hline $06176-1036$ & Red Rectangle & - & $\mathrm{P}$ & - & $\mathrm{B} 9-\mathrm{A} 0^{10)}$ & PAH; crystalline silicate \\
\hline $19163+2745$ & EP Lyr & - & - & RVB & $\mathrm{Fp}^{11)}$ & PAH; crystalline silicate \\
\hline
\end{tabular}

1) Keenan \& Boeshaar (1980); 2) Lloyd Evans (1991); 3) Kwok et al. (1997); 4) Lloyd Evans (1990); 5) Poulakos (1978); 6) Gigoyan \& Mickaelian (2012); 7) Chen et al. (2007); 8) Chan (1993); 9) Drew et al. (2005); 10) Cohen et al. (1975); 11) Preston et al. (1963); 12) MacConnell (2003); 13) Kwok et al. (1997); 14) Aoki, Tsuji, \& Ohnaka (1998)

tion 4.1) may indicate that the silicate dust formation phase in the previous O-rich atmosphere was relatively recent.

There are two objects in subclass 3. They are Crich post-AGB stars with crystalline silicate features. These stars show PAH and crystalline silicate features. HD 44179, the central star of the Red Rectangle (IRAS 06176-1036), is known as a spectroscopic binary with a period of 298 days (Van Winckel et al. 1995). EP Lyr (IRAS 19163+2745) is a post-AGB star which is a RV Tau variable with the period of $\mathrm{P}=83.46$ days (Zsoldos 1995; Gonzalez et al. 1997). RV Tauri stars are luminous evolved stars crossing the instability strip. EP Lyr shows unusually low infrared excess that means a lower dust mass compared to the Red Rectangle.

\subsection{The Silicate Features at $10 \mu \mathrm{m}$}

In order to measure the characteristics of a silicate feature at $10 \mu \mathrm{m}$, we assume that the local continuum is a simple power law as follows:

$$
\lambda F_{\lambda, \text { cont }}=K \lambda^{-p} \text {. }
$$

where the $\lambda F_{\lambda, \text { cont }}$ is the assumed local continuum, $\mathrm{K}$ and $\mathrm{P}$ are constants to determine. The simple power law was fit to the continuum in the $\sim 8 \mu \mathrm{m}$ and $\sim 13.5$ $\mu \mathrm{m}$ region. The used spectral data for the continuum fitting are IRAS LRS or ISO SWS. After the continuum fitting, we obtain the normalized spectra,

$$
S_{\lambda}=\frac{\lambda F_{\lambda, o b s}}{\lambda F_{\lambda, \text { cont }}}
$$

where $\lambda F_{\lambda, o b s}$ is the observed spectrum.

Figure 2 shows the normalized spectra of some SCSs with good quality data. All of the objects are in subclass 2 except S Lyr (subclass 1) and Red Rectangle (subclass 3 ). They show various shapes of the silicate feature at $10 \mu \mathrm{m}$. 

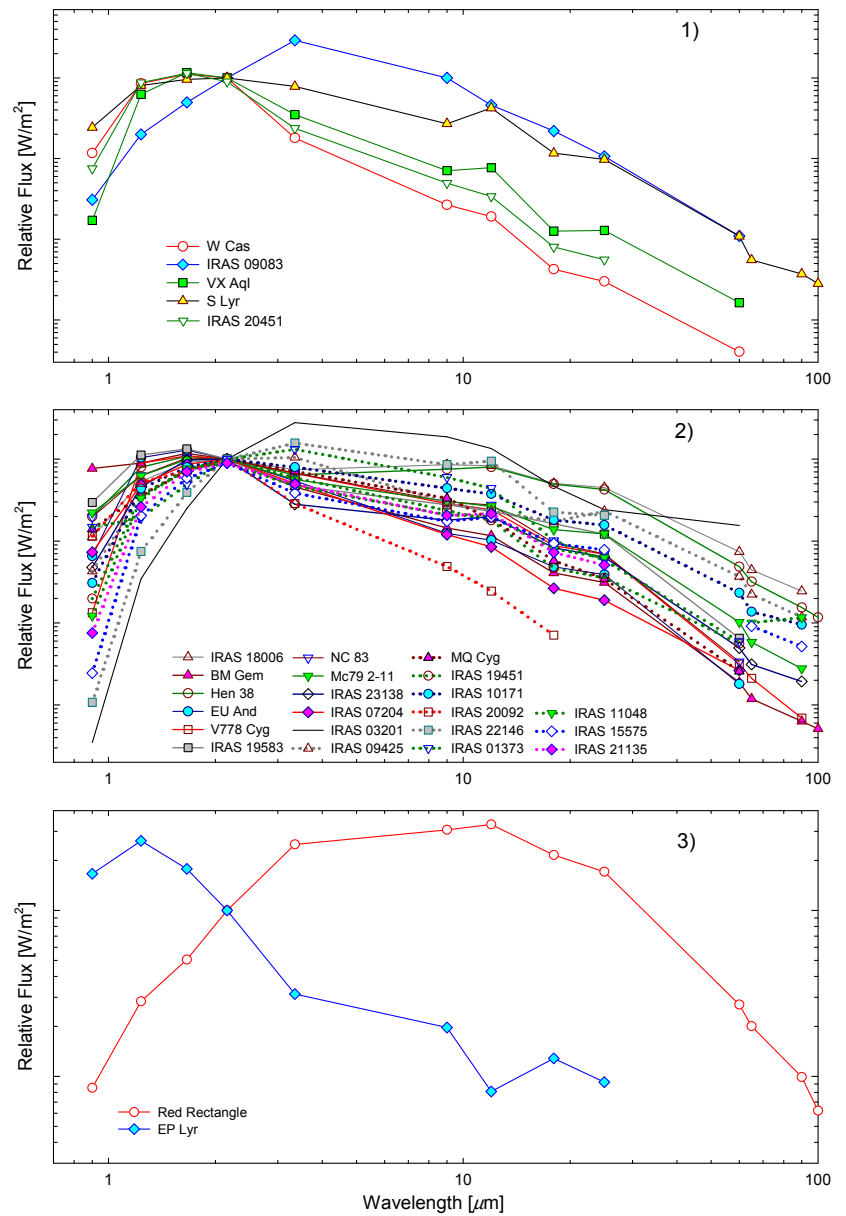

Figure 1. Relative spectral energy distributions for three subclasses. 1) SC/CS stars with silicate emission features; 2) carbon stars with silicate features; 3) C-rich post-AGB stars with crystalline silicate

For S Lyr (subclass 1), the $10 \mu \mathrm{m}$ feature is very weak. For Red Rectangle (subclass 3), no evident silicate feature at $10 \mu \mathrm{m}$ is seen but PAH features at 7.7, 8.6 and $11.3 \mu \mathrm{m}$ as well as crystalline silicate features at longer wavelengths are evident.

For most of the objects in subclass 2, the silicate feature at $10 \mu \mathrm{m}$ is easily detectable. The dominant feature appears in two objects: V778 Cyg and BM Gem. The $10 \mu \mathrm{m}$ amorphous silicate feature is less prominent for Hen 38 and IRAS $23138+6204$. The feature is weak and flat for IRAS 09425-6040, IRAS 10170-6205. The $10 \mu \mathrm{m}$ absorption feature of amorphous silicate is identified for IRAS $03201+5459$.

The $11.3 \mu \mathrm{m}$ emission feature seen in spectra of IRAS 03201+5459 and 09425-6040 (subclass 2) can be caused by $\mathrm{SiC}, \mathrm{PAH}$ or crystalline forsterite. However, $11.3 \mu \mathrm{m}$ emission features due to PAH or crystalline forsterite are narrower and involve other features at other wavelengths. There is no other conspicuous features indicating PAH or crystalline silicate in the spectra of IRAS $03201+5459$ and 09425-6040. Therefore, the emission feature at $11.3 \mu \mathrm{m}$ is likely to be caused by $\mathrm{SiC}$ grains.

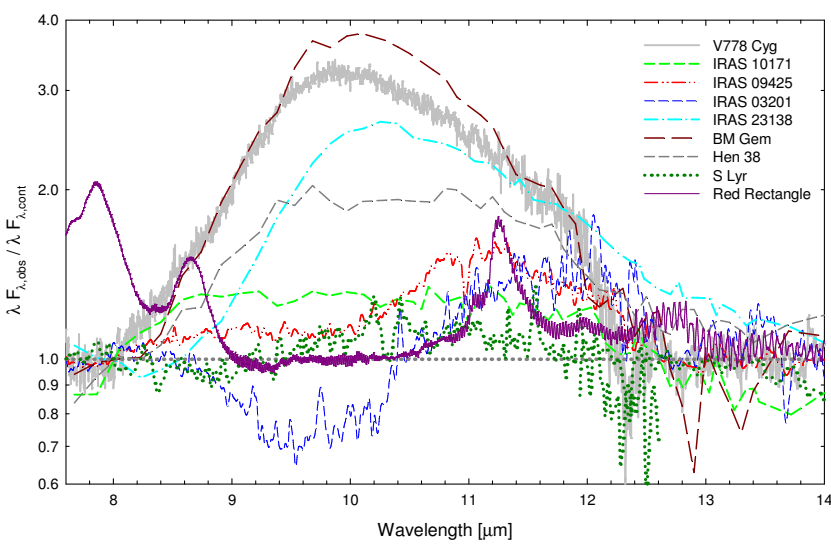

Figure 2. Profile lines of the observed $10 \mu \mathrm{m}$ silicate feature.

For some of the SCSs in subclass 2 (e.g., IRAS 101716205 and Hen 38), the silicate feature at $10 \mu \mathrm{m}$ is weak and flat. Kessler-Silacci et al. (2006) argued the weak and flat features of amorphous silicate can be interpreted as grain growth (from 0.1 to $1.0 \mu \mathrm{m}$ in radius). The unusual silicate features in the SCSs indicate that the dust grains experience significant chemical and physical processing in their environment. Generally, a suitable site for grain growth is believed to be a stationary disk rather than an expanding shell. The weak and flat silicate feature may imply dust processing in a long lived reservoir such as a disk.

\section{Dust Shell Models for the Chemical Transition Phase}

To investigate the effect of the chemical transition phase from $\mathrm{O}$ to $\mathrm{C}$, we use simple radiative transfer models for the detached silicate dust shells. The spectral energy distributions and infrared color-color diagrams of SCSs are compared with the theoretical model results.

\subsection{Radiative Transfer Model Calculations}

In this work, we use the radiative transfer code RADMC-3D (http://www.ita.uni-heidelberg.de/ dullemond/software/radmc-3d/) to calculate the model SEDs. RADMC-3D is the three-dimensional version of the code for investigating dust continuum radiative transfer processes based on the Monte Carlo simulation method of Bjorkman \& Wood (2001). For this work, we consider the geometry of one-dimensional grid only. Given spherically symmetric density structures, the code calculates the dust temperature and scattering source function at every geometric point in the model. Model SEDs can be calculated by integrating the intensity inside synthetic observing apertures at each wavelength.

\subsection{Basic Model Parameters}

In this paper, we assume a spherically symmetric dust shell around a single star. We use similar schemes for dust density distribution as those used by Suh \& Kwon 
(2013). Although AGB stars are pulsating, the overall continuous density distribution is believed to be maintained for a time scale larger than the pulsation period (e.g., Suh 1999; Suh 2004).

In this paper, we assume that the dust density distribution is continuous $\left(\rho \propto r^{-2}\right)$ from the inner radius $\left(R_{\text {in }}\right)$ to the outer radius $\left(R_{\text {out }}\right)$ for each dust shell. For the dust density distribution of a spherically symmetric dust shell, we use the simple power law equation

$$
\rho(r)=\beta \rho_{i n}\left(r / R_{i n}\right)^{-2},
$$

where $\rho_{i n}$ is the density at $R_{\text {in }}$ of the dust shell.

The inner shell dust temperature $\left(T_{c}\right)$ is the dust temperature at $R_{i n}$. In model calculations, we may find $R_{i n}$ at which the dust temperature $\left(T_{c}\right)$ becomes the desired one after a few trials. The model SEDs are sensitively dependent on $T_{c}$. We may roughly regard the temperature $T_{c}$ as the dust formation (or condensation) temperature. However, $T_{c}$ is not necessarily the same as the dust formation temperature, depending on the physical conditions of pulsating $\mathrm{OH} / \mathrm{IR}$ stars (see Suh 2004).

For the central star, we assume that the luminosity is $1 \times 10^{4} L_{\odot}$ and a stellar blackbody temperature is $2500 \mathrm{~K}$.

\subsection{The Model Setup}

We investigate the time scales for the appearance of the silicate feature after the chemical transition of the central star from $\mathrm{O}$ to $\mathrm{C}$ which is characterized by a sudden cessation of the O-rich mass-loss. Because the central star does not provide O-rich material after the chemical transition, the silicate dust shell will be detached gradually from the central star and the silicate dust features will become weaker. The transition object with a C-rich central star can be classified to be a SCS until the silicate dust feature completely disappears. Previously estimated time scale for the transitional object as a SCS is predicted to last only a few decades (e.g., Little-Marenin et al. 1988).

The initial inner radius $\left(R_{0}\right)$ of dust shell is chosen by the radius where the dust temperature is $1000 \mathrm{~K}$. As the dust shell expands, the inner radius $\left(\mathrm{R}_{i n}\right)$ of the dust shell increases with time $\left(R_{\text {in }}=R_{0}+V_{\text {exp }} t\right)$ after the chemical transition begins (or the O-rich mass-loss stops at $\left.R_{0}\right)$.

We use spherically symmetric O-rich dust shells with four different dust optical depths of $\tau_{10, \text { int }}=0.01,0.1,1$ and 7 as the initial conditions. For all of the models, we assume that the stellar wind velocity $V_{\text {exp }}$ is constant. We use the dust opacity functions of warm $\left(\tau_{10, \text { int }}=\right.$ $0.01,0.1$ and 1$)$ and cold $\left(\tau_{10, \text { int }}=7\right)$ amorphous silicate derived by Suh (1999). We assume that all dust grains are spherical with a uniform radius of $0.1 \mu \mathrm{m}$ and the scattering is assumed to be isotropic.

For each model, the dust temperature $\left(\mathrm{T}_{c}\right)$ at the $\mathrm{R}_{\text {in }}$ decreases as the O-rich dust shell expands. We fixed the outer radius $\left(\mathrm{R}_{\text {out }}\right)$ of the dust shell to $10^{5} \mathrm{AU}(\sim 0.5$ $\mathrm{pc})$. For the all of the models, dust temperatures at
$\mathrm{R}_{\text {out }}$ are lower than $30 \mathrm{~K}$ which is comparable to the temperature of interstellar medium.

\subsection{Evolution of the Silicate Feature}

In Figure 3, we present the profile lines of the silicate feature at $10 \mu \mathrm{m}$ obtained from the model SEDs of the transitional dust shells explained in Section 5.3. As explained in Section 3.1, the normalized model spectra are obtained using the local continuum Equation (1). As the dust shell expands, the strength of the silicate emission feature becomes weak and the peak of the feature moves slightly to longer wavelengths. We may claim that the silicate feature disappears when the following condition is satisfied around $10 \mu \mathrm{m}$ :

$$
\frac{F_{c a l, \lambda}}{F_{c o n t, \lambda}}-1<0.05
$$

where $F_{c a l, \lambda}$ is the calculated flux. We find that the silicate emission feature for the expanding O-rich shell is retained only for a short time and fade away rapidly. We assume that the shell expands outward with a constant velocity of $10 \mathrm{~km} / \mathrm{sec}$ (e.g., Loup et al. 1993) thereby moving $210 \mathrm{AU}$ in 100 years. The life time scales for the silicate emission feature for the models of $\tau_{10, \text { int }}=0.01,0.1,1$ and 7 are about 50, 140, 380 and 400 years, respectively.

As the O-rich dust shell expands, silicate emission features disappear due to fast dilution of silicate dust density and/or cooling of dust temperature $(<150 \mathrm{~K})$ (See Table 4$)$. For the model of $\tau_{10, \text { int }}=7$, the silicate absorption feature at $10 \mu \mathrm{m}$ is retained only for a short time scale ( $\sim 28$ years). Then the silicate emission feature lasts for about 380 years.

Though the transition models reproduce various shapes of the silicate feature, they can not reproduce the flat silicate features seen in some of the SCSs (e.g., IRAS 10171-6205). Other theoretical models (e.g., a disk model) could be more useful.

\section{Two-Color Diagrams}

We present the IRAS 2CDs for AGB stars using [25][60] versus [12]-[25] in Figure 4 and the NIR-IRAS 2CD using [12]-[25] versus $K-L$ in Figure 5. See Suh \& Kwon (2011) for detailed explanations about the color indices. We use available NIR data in $K$ and $L$ bands obtained from ground-based observations as explained in Suh \& Kwon 2011. We plot the observed colors of 29 SCSs verified in this paper and 15 unconfirmed objects and 12 candidate objects presented by CS11. We also plot the observed colors of 3373 O-rich AGB stars listed in Kwon \& Suh (2012) and 1168 C-rich AGB stars for comparison.

For any $2 \mathrm{CD}$, we use only objects with a good photometric quality flag $(q=3)$ to plot at any wavelength. We also plot the results of model calculations for the spherical dust shell models presented in Section 5 for comparison.

Each model track on the 2CD is composed of 24 points of different inner radii of dust shell from $r_{1}$ to 

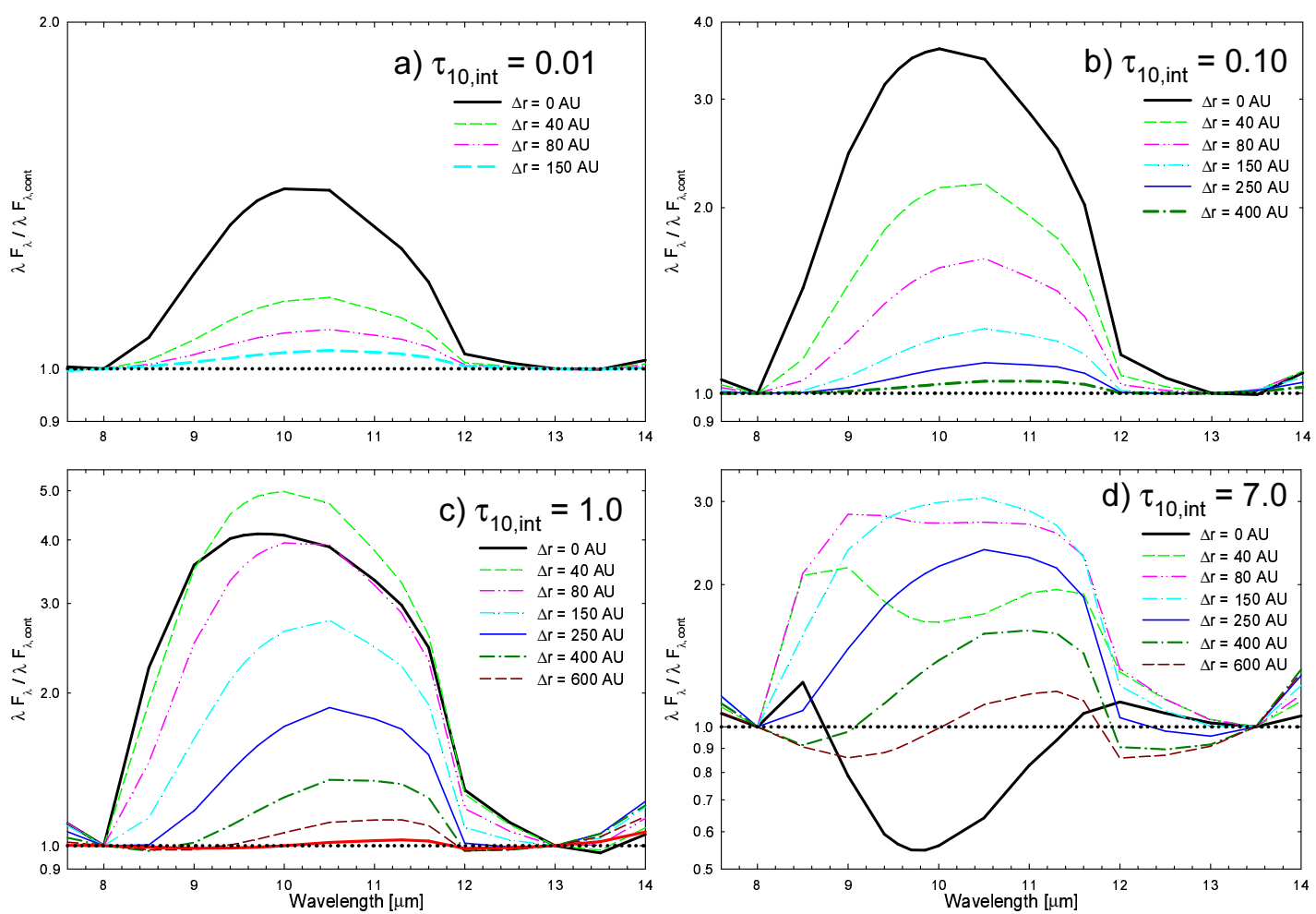

Figure 3. Profile lines of the silicate $10 \mu \mathrm{m}$ feature for the transitional models.

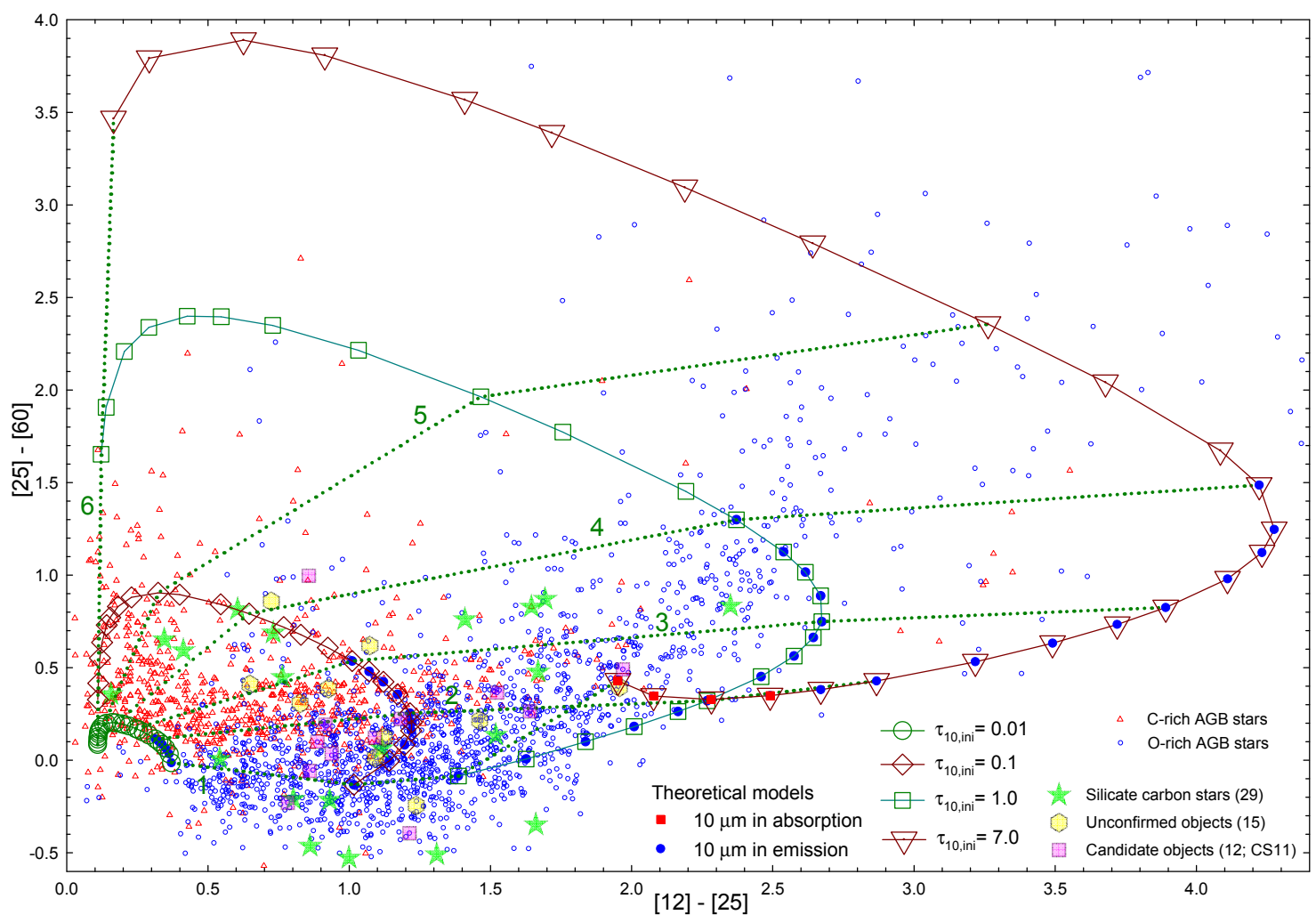

Figure 4. The IRAS 2CD ([25]-[60] versus [12]-[25]). 


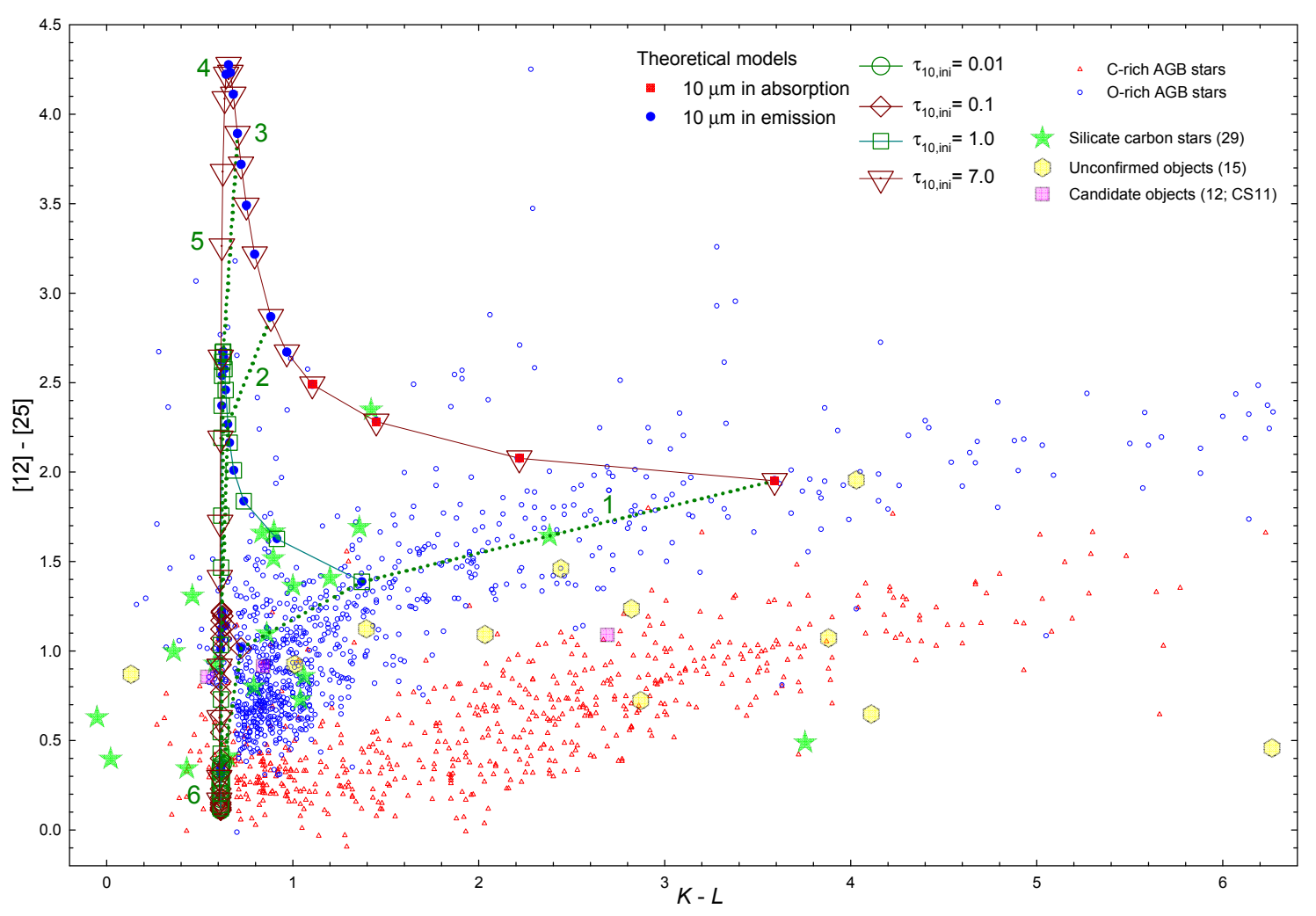

Figure 5. The NIR-IRAS 2CD ([12]-[25] versus $K-L)$.

$r_{1}+15000$ AU. The amount of shell detachment $(\Delta r=$ $\left.V_{\text {exp }} t\right)$ corresponds to the time steps from 0 to about 10000 years under the condition of $V_{\text {exp }}=10 \mathrm{~km} / \mathrm{s}$. We examine the shape of the silicate feature at $10 \mu \mathrm{m}$ to determine the silicate emission or absorption phases as explained in Section 5.4. We represent the silicate absorption phases by red filed squares and the emission phases by blue filled cicles on the 2CDs.

In Table 5, we list the model parameters of $\tau_{10}=$ $0.01,0.1,1$ and 7 for six time step points which are also labeled in the $2 \mathrm{CDs}$. The inner radius $\left(\mathrm{R}_{i n}\right)$ of the dust shell, dust density $\left(\rho_{i n}\right)$ at the inner radius, dust temperature $\left(\mathrm{T}_{c}\right)$ at the inner radius and $\tau_{10}$ of each model are listed. The points labeled with number 1 indicate the initial points for normal O-rich AGB stars. The points labeled as 2, 3 and 4 refer to the end point of silicate emission phase for the model of $\tau_{10}=0.01,0.1$ and 1 , respectively. The points 5 and 6 are the model points for the expansion times of $\sim 1000$ and $\sim 10^{4}$ years, respectively.

The loop-like shape of evolutionary tracks on the [25]-[60] versus [12]-[25] diagram (Figure 4) resemble the works of Willems \& de Jong (1988) and Chan \& Kwok (1988). The model tracks show that the silicate emission feature remains toward higher values of [12]-[25] color but disappears for higher values of [25][60] color. Although some SCSs are located around the model track on the [25]-[60] versus [12]-[25] diagram, the model can not cover the wide distributions of ob- served SCSs. Although it takes less time to show silicate features in the transition model, the expanding shell can contribute to far-IR continuum emission for a much longer time scale. As we can see in Figure 4, the flux excesses at $60 \mu \mathrm{m}$ relative to O-rich AGB stars are due to the cooling dust remnants ejected at least $\sim 100$ and 1000 years ago for the models of $\tau_{10}=0.01$ and 7 , respectively. These model tracks for later stages are particularly successful in explaining the SCSs in the upper-left region of the $2 \mathrm{CD}$ where optical carbon stars are located.

In the [12]-[25] versus $K-L$ diagram (Figure 5), the model tracks develop toward the upper left region to converge quickly (less than 500 years) to the vertical line of $K-L \simeq 0.6$. Many of the SCSs are outside the regions of the transition model tracks. This is because carbon stars show strong photospheric molecular bands in $K$ and $L$ which can skew the $K-L$ color value away from what is expected for a blackbody of $2500 \mathrm{~K}$. Note that the time scale for the model tracks is relatively short ( $\sim 1000$ years from the label point 1 to point 5$)$.

Contrary to previous works (Willems \& de Jong 1988; Chan \& Kwok 1988), our results show that the life time of the silicate feature can be much longer (up to 400 years depending on the initial dust optical depth; see Section 5.4). This is because we use higher initial dust optical depths $\left(\tau_{10}=1\right.$ and 7$)$ which are appropriate for some observed SCSs (e.g., the IRAS 03201+5459 shows the $10 \mu \mathrm{m}$ silicate feature in absorption) as well 
Table 5

Parameters for the transitional dust shell models

\begin{tabular}{cccccccc}
\hline$\tau_{10, \text { int }}$ & Parameters & 1 & 2 (47 years) & 3 (142 years $)$ & 4 (400 years $)$ & $5\left(\sim 10^{3}\right.$ years $)$ & $6\left(\sim 10^{4}\right.$ years $)$ \\
\hline 0.01 & $\mathrm{R}_{\text {in }}[\mathrm{AU}]$ & 12.9 & 116.4 & 312.6 & 839 & 2059 & 20321 \\
& $\rho_{\text {in }}\left[\mathrm{g} / \mathrm{cm}^{3}\right]$ & $2.75 \mathrm{E}-20$ & $3.38 \mathrm{E}-22$ & $4.69 \mathrm{E}-23$ & $6.51 \mathrm{E}-24$ & $1.08 \mathrm{E}-24$ & $1.11 \mathrm{E}-26$ \\
& $\mathrm{~T}_{c}[\mathrm{~K}]$ & 1001 & 324 & 207 & 140 & 100 & 46 \\
& $\tau_{10}$ & 0.01 & $1.1 \mathrm{E}-3$ & $4.1 \mathrm{E}-4$ & $1.5 \mathrm{E}-4$ & $6.1 \mathrm{E}-5$ & $5.0 \mathrm{E}-6$ \\
& Silicate $^{*}$ & Emission & Emission & No & No & No & No \\
\hline 0.1 & $\mathrm{R}_{\text {in }}[\mathrm{AU}]$ & 13.4 & 113.8 & 322.8 & 829 & 2027 & 20461 \\
& $\rho_{\text {in }}\left[\mathrm{g} / \mathrm{cm}^{3}\right]$ & $2.64 \mathrm{E}-19$ & $3.69 \mathrm{E}-21$ & $4.58 \mathrm{E}-22$ & $6.94 \mathrm{E}-23$ & $1.16 \mathrm{E}-23$ & $1.14 \mathrm{E}-25$ \\
& $\mathrm{~T}_{c}[\mathrm{~K}]$ & 1001 & 329 & 205 & 141 & 101 & 46 \\
& $\tau_{10}$ & 0.1 & 0.012 & $4.2 \mathrm{E}-3$ & $1.6 \mathrm{E}-3$ & $6.5 \mathrm{E}-4$ & $5.2 \mathrm{E}-5$ \\
& Silicate $^{*}$ & Emission & Emission & Emission & No & No & No \\
\hline 1.0 & $\mathrm{R}_{\text {in }}[\mathrm{AU}]$ & 15.7 & 118.6 & 325.5 & 818 & 2056 & 20150 \\
& $\rho_{\text {in }}\left[\mathrm{g} / \mathrm{cm}^{3}\right]$ & $2.25 \mathrm{E}-18$ & $3.97 \mathrm{E}-20$ & $5.28 \mathrm{E}-21$ & $8.35 \mathrm{E}-22$ & $1.32 \mathrm{E}-22$ & $2.33 \mathrm{E}-24$ \\
& $\mathrm{~T}_{c}[\mathrm{~K}]$ & 999 & 331 & 206 & 142 & 100 & 46 \\
& $\tau_{10}$ & 1.0 & 0.13 & 0.048 & 0.019 & $7.5 \mathrm{E}-3$ & $6.2 \mathrm{E}-4$ \\
& Silicate $^{*}$ & Emission & Emission & Emission & Emission & No & No \\
\hline 7.0 & $\mathrm{R}_{\text {in }}[\mathrm{AU}]$ & 18.3 & 122.1 & 329.2 & 851 & 2105 & 21621 \\
& $\rho_{\text {in }}\left[\mathrm{g} / \mathrm{cm}^{3}\right]$ & $1.22 \mathrm{E}-17$ & $2.74 \mathrm{E}-19$ & $3.77 \mathrm{E}-20$ & $5.64 \mathrm{E}-21$ & $9.22 \mathrm{E}-22$ & $8.73 \mathrm{E}-24$ \\
& $\mathrm{~T}_{c}[\mathrm{~K}]$ & 1000 & 336 & 167 & 130 & 93 & 43 \\
& $\tau_{10}$ & 7.0 & 1.05 & 0.351 & 0.136 & 0.0549 & $5.34 \mathrm{E}-3$ \\
& Silicate $^{*}$ & Absorption & Emission & Emission & Emission & No & No \\
\hline
\end{tabular}

${ }^{*}$ The silicate feature at $10 \mu \mathrm{m}$.

as smaller ones $\left(\tau_{10}=0.01\right.$ and 0.1$)$. The previous works considered only the SCSs with the initial $10 \mu \mathrm{m}$ silicate feature in emission $\left(\tau_{10}<1\right)$.

\section{Discussion}

As explained in Sections 5.4 and 6, we find that the detached silicate dust shell models can not explain the observed SEDs for the whole sample of SCSs. We may need a more complicated geometry for the dust distribution. A more sophisticated theoretical model using a circumbinary or circumstellar dust disk as well as the dust shell would be more useful.

There are some observational evidences for the presence of a disk in some SCSs. For BM Gem and EU And, the variations of radial velocity are detected due to motions in a binary system by Barnbaum et al. (1991). And Kahane et al. (1998) and Jura \& Kahane (1999) observed the narrow CO emission lines supporting the presence of long-lived reservoirs of orbiting gas in the two stars. Izumiura et al. (2008) found the P Cygnitype profile in the violet spectra of BM Gem which indicates the presence of an outflow with at least $400 \mathrm{~km} / \mathrm{s}$. Ohnaka et al. (2008) made mid-IR interferometry observations toward BM Gem which suggested a circumcompanion disk. And VLBA $\mathrm{H}_{2} \mathrm{O}$ maser observations toward EU And supported the circum-companion disk (Ohnaka \& Boboltz 2008). For V778 Cyg, Szczerba et al. (2006) obtained high resolution $\mathrm{H}_{2} \mathrm{O}$ maser maps at $22 \mathrm{GHz}$ and they concluded that the object is composed of a C-rich star and a companion that stores an O-rich disk. Those observations are strong evidences for presence of an O-rich disk around a SCS which is actually a binary system. We expect that most SCS are likely to be binary systems rather than single stars.

\section{SUMMARY}

We have collected 44 SCSs from the literature and verified their classification. We have confirmed that 29 objects are real SCSs and 15 objects are unconfirmed. For some objects, it is doubtful whether the central star is a carbon star. Other objects seem to be contaminated by interstellar clouds.

For the confirmed 29 SCSs, we have classified them into three subclasses based on the evolution phase of the central star: SC/CS stars with silicate emission features, carbon stars with silicate features and C-rich post-AGB stars with crystalline silicate. The shapes of the silicate emission feature at $10 \mu \mathrm{m}$ for various SCSs have been investigated.

We have made radiative transfer model calculations for the transitional dust shell models of SCSs with detached O-rich dust shells. We have compared the theoretical model results with the observations of SCSs on different 2CDs. The life time of the silicate emission feature at $10 \mu \mathrm{m}$ has been estimated to be $50-400$ years depending on the thickness of the dust shell in the Orich phase. For model of $\tau_{10, i n i}=7$, silicate absorption phase appears only for a short time scale.

The transitional dust shell models presented in this paper may provide only a partial explanation for the origin of SCSs because the models do not reproduce all of the observations. For the chemical transition model without considering the effect of a disk, the life time of the silicate feature is too short. We expect that a more sophisticated theoretical model using a circumbinary or circumstellar dust disk as well as the dust shell would be useful. 


\section{ACKnOWLedgments}

This work was supported by the research grant of the Chungbuk National University in 2013. K.-W. Suh was supported by Basic Science Research Program through the National Research Foundation of Korea (NRF) funded by the Ministry of Science, ICT \& Future Planning (NRF-2013R1A1A2057841).

\section{REFERENCES}

Aaronson, M., Blanco, V. M., Cook, K. H., et al. 1989, Southern Milky Way Carbon Stars - New Candidates, JHK Photometry, and Radial Velocities, ApJS, 70, 637

Alksnis, A., Balklavs, A., Dzervitis, U., et al. 2001, General Catalog of galactic Carbon stars, 3d Ed., Balt. Astron., 10,1

Aoki, W., Tsuji, T., \& Ohnaka, K. 1998, Infrared Spectra of Carbon Stars Observed by the ISO SWS I. Molecular Absorption in N-Type and SC-Type Stars, A\&A, 340, 222

Barnbaum, C., Kastner, J. H., Morris, M., et al. 1991, Carbon Stars Associated with Oxygen-Rich Circumstellar Envelopes - EU Andromedae, BM Geminorum and V 778 Cygni, A\&A, 251, 79

Bjorkman, J. E., \& Wood, K. 2001, Radiative Equilibrium and Temperature Correction in Monte Carlo Radiation Transfer, ApJ, 554, 615

Busso, M., Gallino, R., \& Wasserburg, G. J. 1999, Nucleosynthesis in Asymptotic Giant Branch Stars: Relevance for Galactic Enrichment and Solar System Formation, ARAA, 37, 239

Chan, S. J. 1993, Spectroscopic Study of Carbon Stars with Silicate Features. 1: Observations, AJ, 106, 2126

Chan, S. J., \& Kwok, S. 1988, The Transition from OxygenRich to Carbon Stars, ApJ, 334, 362

Chan, S. J., \& Kwok, S. 1990, Evolution of Infrared Carbon Stars, A\&A, 237, 354

Chan, S. J., \& Kwok, S. 1991, New Candidates for Carbon Stars with Silicate Features, ApJ, 383, 837

Chen, P.-S., \& Yang, X.-H., Zhang, P. 2007, Infrared Study of J-Type Carbon Stars Based on Infrared Astronomical Satellite, Two Micron All Sky Survey, and Infrared Space Observatory Data, AJ, 134, 214

Chen, P.-S., \& Shan, H.-G. 2011, Identification of New Silicate Carbon Stars, ApSS, 335, 457

Cohen, M., Anderson, C. M., Cowley, A., et al. 1975, The Peculiar Object HD 44179 'The Red Rectangle', ApJ, 196, 179

Cutri, R. M., Skrutskie, M. F., Van Dyk, S., et al. 2003, The IRSA 2MASS All-Sky Catalog of Point Sources. In: NASA/IPAC Infrared Science Archive

Drew, J. E., Greimel, R., \& Irwin, M. J. 2005, The INT Photometric H Survey of the Northern Galactic Plane (IPHAS), MNRAS, 362, 753

Egan, M. P., Price, S. D., \& Kraemer, K. E. 2003, The Midcourse Space Experiment Point Source Catalog Version 2.3, BAAS, 203, 5708

Engels, D. 1994, More H2O Maser in J-Type Carbon Stars, A\&A, 285, 497

Epchtein, N., Le Bertre, T., Lepine, J. R. D., et al. 1987, Valinhos 2.2 Micron Survey of the Southern Galactic Plane. II - Near-IR Photometry, IRAS Identifications and Nature of the Sources, A\&AS, 71, 39

Epchtein, N., Le Bertre, T., \& Lepine, J. R. D. 1990, Carbon Star Envelopes - Near-IR Photometry, Mass Loss and
Evolutionary Status of a Sample of IRAS Stars, A\&A, 227,82

Fouque, P., Le Bertre, T., \& Epchtein, N. 1992, NearInfrared Photometry of a Sample of IRAS Point Sources, A\&AS, 93, 151

Gielen, C., Van Winckel, H., Min, M., et al. 2008, SPITZER Survey of Dust Grain Processing in Stable Discs around Binary Post-AGB Stars, A\&A, 490, 725

Gielen, C., Van Winckel, H., Matsuura, M., et al. 2009, Analysis of the Infrared Spectra of the Peculiar Post-AGB Stars EP Lyrae and HD 52961, A\&AS, 503, 843

Gigoyan, K. S., \& Mickaelian, A. M. 2012, Revised and Updated Catalogue of the First Byurakan Survey of LateType Stars, MNRAS, 419, 3346

Gonzalez, G., Lambert, D. L., \& Giridhar, S. 1997, Abundance Analyses of the Field RV Tauri Variables: EP Lyrae, DY Orionis, AR Puppis, and R Sagittae, ApJ, 479,427

Groenewegen, M. A. T., van den Hoek, L. B., \& de Jong, T. 1995, The Evolution of Galactic Carbon Stars., A\&A, 293,381

Groenewegen, M. A. T., Sevenster, M., Spoon, H. W. W., et al. 2002, Millimetre Observations of Infrared Carbon Stars. I. The data, A\&A, 390, 501

Guglielmo, F., Epchtein, N., Le Bertre, T., et al. 1993, Identification of 106 New Infrared Carbon Stars in the IRAS Point Source Catalog - Near-Infrared Photometry and Their Space Distribution in the Galaxy, A\&AS, 99, 31

Guglielmo, F., Epchtein, N., Arditti, F., et al. 1997, New Infrared Carbon Stars in the IRAS Point Source Catalog., A\&AS, 122, 489

Iben, Jr. I. 1981, The Carbon Star Mystery - Why Do the Low Mass Ones Become Such, and Where Have All the High Mass Ones Gone, ApJ, 246, 278

Izumiura, H., Noguchi, K., Aoki, W., et al. 2008, Evidence for a Companion to BM Gem, a Silicate Carbon Star, ApJ, 682, 499

Jiang, B. W., Deguchi, S., Hu, J. Y., et al. 1997, Identification of IRAS Sources in the Outer Disk of the Galaxy, AJ, 113, 1315

Jura, M., \& Kahane, C. 1999, Orbiting Molecular Reservoirs around Evolved Red Giant Stars, ApJ, 521, 302

Kahane, C., Barnbaum, C., Uchida, K., et al. 1998, A Circumbinary Reservoir around BM Geminorum?, ApJ, 500, 466

Keenan, P. C., \& Boeshaar, P. C. 1980, Spectral Types of S and SC Stars on the Revised MK System, ApJS, 43, 379

Kessler-Silacci, J., Augereau, J., Dullemond, C. P., et al. 2006, c2d Spitzer IRS Spectra of Disks around T Tauri Stars. I. Silicate Emission and Grain Growth, A\&A, 639, 275

Kwok, S., Volk, K., \& Bidelman, W. P. 1997, Classification and Identification of IRAS Sources with Low-Resolution Spectra, ApJS, 112, 557

Kwon, Y.-J., \& Suh, K.-W. 2012, Properties of OH, SiO, and H2O Maser Emission in O-Rich AGB Stars, JKAS, 45, 139

Little-Marenin, I. R. 1986, Carbon Stars with Silicate Dust in Their Circumstellar Shells, ApJL, 307, L15

Little-Marenin, I. R., Benson, P. J., \& Dickinson, D. F. 1988, Masers Associated with Two Carbon Stars - V778 Cygni and EU Andromedae, ApJ, 330, 828

Lloyd Evans, T. 1990, Carbon Stars with Silicate Dust Shells. I - Carbon Stars with Enhanced C-13 (J Stars), 
MNRAS, 243, 336

Lloyd Evans, T. 1991, Carbon Stars with Silicate Dust Shells. II - More Stars with Enhanced C-13 (J Stars), MNRAS, 249, 409

Loup, C., Forveille, T., Omont, A., \& Paul, J. F. 1993, $\mathrm{CO}$ and HCN Observations of Circumstellar Envelopes. A Catalogue - Mass Loss Rates and Distributions, A\&AS, 99, 291

MacConnell, D. J. 2000, New S Stars Found in a Southern Galactic Plane Survey, PASP, 112, 65

MacConnell, D. J. 2003, Southern Cool Carbon Stars Found on Near-Infrared Objective Prism Plates, PASP, 115, 351

MacConnell, D. J. 2006, Southern Cool Stars Misclassified as Carbon Stars, Inf. Bull. Var. Stars, 5671, 1

Molster, F. J., Yamamura, I., Waters, L. B. F. M., et al. 2001, IRAS 09425-6040: A Carbon Star Surrounded by Highly Crystalline Silicate Dust, A\&A, 366, 923

Monet, D. G., Levine, S. E., Canzian, B., et al. 2003, The USNO-B Catalog, AJ, 125, 984

Morris, M. 1987, Mechanisms for Mass Loss from Cool Stars, PASP, 99, 1115

Murakami, H., Baba, H., Barthel, P., et al. 2007, The Infrared Astronomical Mission AKARI, PASJ, 59, 369

Ohnaka, K., \& Boboltz, D. A. 2008, Imaging the OxygenRich Disk toward the Silicate Carbon Star EU Andromedae, A\&A, 478, 809

Ohnaka, K., Izumiura, H., Leinert, C., et al. 2008, Asymmetric Silicate Dust Distribution toward the Silicate Carbon Star BM Geminorum, A\&A, 490, 173

Poulakos, C. 1978, Extremely Red Stars at $\mathrm{e}^{I I} \approx 113^{\circ}$, $10^{\circ} \leq \mathrm{b}^{I I} \leq 90^{\circ}, \mathrm{A} \& \mathrm{AS}, 32,395$

Preston, G. W., Krzeminski, W., Smak, J., et al. 1963, A Spectroscopic and Photoelectric Survey of the RV Tauri Stars, ApJ, 137, 401

Samus, N. N., Durlevich, O. V., Kazarovets, E. V., Kireeva, N. N., Pastukhova, E. N., \& Zharova, A. V. 2011, General Catalog of Variable Stars (GCVS database, Version 2011Jan), VizieR online data catalogue, B/GCVS

Skiff, B. A. 2014, General Catalogue of Stellar Spectral Classifications (Version 2013-Jul), VizieR B/mk

Suh, K.-W. 1999, Optical Properties of the Silicate Dust Grains in the Envelopes around Asymptotic Giant Branch Stars, MNRAS, 304, 389

Suh, K.-W. 2000, Optical Properties of the Carbon Dust Grains in the Envelopes around Asymptotic Giant Branch Stars, MNRAS, 315, 740

Suh, K.-W. 2004, Pulsation Phase-Dependent Dust Shell Models for Oxygen-rich Asymptotic Giant Branch Stars, ApJ, 615, 485

Suh, K.-W., \& Kwon, Y.-J. 2011, Infrared Two-Colour Diagrams for AGB Stars Using AKARI, MSX, IRAS and Near-Infrared Data, MNRAS, 417, 3047

Suh, K.-W., \& Kwon, Y.-J. 2013, Water Ice in High MassLoss Rate OH/IR Stars, ApJ, 762, 113

Szczerba, R., Szymczak, M., Babkovskaia, N., et al. 2006, Oxygen-Rich Disk in the V778 Cygni System Resolved, A\&A, 452, 561

te Lintel Hekkert, P. 1991, An OH Survey of Very Cold IRAS Point Sources, A\&A, 248, 209

Van Winckel, H., Waelkens, C., \& Waters, L. B. F. M. 1995, The Extremely Iron-Deficient "Post-AGB" Stars and Binaries., A\&A, 293, 25

Willems, F. J., \& de Jong, T. 1986, Carbon Stars with Oxygen-Rich Circumstellar Dust Shells Observational Evidence for the Onset of the Carbon Star Phase, ApJL,
309, 39

Willems, F. J., \& de Jong, T. 1988, IRAS Low Resolution Spectra of Cool Carbon Stars. IV - A Scenario for Carbon Star Evolution, A\&A, 196, 173

Wright, E. L., Eisenhardt, P. R. M., Mainzer, A. K., et al. 2010, The Wide-Field Infrared Survey Explorer (WISE): Mission Description and Initial On-Orbit Performance, AJ, 140, 1868

Yamamura, I., Dominik, C., de Jong, T., et al. 2000, The Origin of Silicate Carbon Stars: ISO/SWS Observation of V778 Cygni, A\&A, 363, 629

Zsoldos, E. 1995, Photometry of EP Lyrae and Period Changes in RV Tauri Stars, A\&A, 296, 122 